\title{
Metropolis"Ant Tribe" Physical Health Status Investigation Taking Beijing City as an Example
}

\author{
Qi LIU \\ Beijing University of Agriculture, Beijing, China
}

\begin{abstract}
Ant tribe" also known as the university graduates in low-income groups, is unable to find work or very low income and live in the metropolis fringe of college students after graduation from the university. Research is to understand the "population health change ant" situation, grasp their group characteristics of physical fitness and health status, provide enhanced physique, promote health sports intervention measures.

1 object and method of study

Research mainly carries on the investigation and research work in Beijing City, $550 \mathrm{low}$ income metropolis and peri-metropolis rural settlements of university graduates with physical measurement method and questionnaire survey method.

Height, weight, vital capacity index, step test, grip strength, vertical jump, sit-ups, push-ups, sit-and-reach, eyes closed, standing on one foot and choice reaction time.
\end{abstract}

2 the results

2.1 body shape

"Ant tribe" male average height of 169.7 centimeters, a standard deviation of 6.15 women; average height is $156.6 \mathrm{~cm}$, the standard deviation of 5.69. The male weighs 64 kilos, standard deviation 11.74; women's weight is 54 kilograms, the standard deviation of 7.38.

\section{2 physical function}

"Ant tribe" male average vital capacity of $3644.9 \mathrm{ml}$, standard deviation 646. 48; average female vital capacity of $2306.1 \mathrm{ml}$, the standard deviation of 684. 77. Test index of average level in male was 50.9, standard deviation 7.34 mean step test index; female 53.6, standard deviation 9.58.

2.3 physical qualities

"Ant tribe" male average grip strength of $44.1 \mathrm{~kg}$, a standard deviation of 5.74 women; average grip strength of $24.3 \mathrm{~kg}$, a st andard deviation of 5.03. The average male vertical jump of $36.6 \mathrm{~cm}$, the standard deviation of 6.45 ; the average woman jump $21.1 \mathrm{~cm}$, the standard deviation of 4.84. Male body anteflexion in sitting position with an average of $7.6 \mathrm{~cm}$, the standard deviation of 7.41 ; female body anteflexion in sitting position with an average of $10 \mathrm{~cm}$, the standard deviation of 5.78.

"Ant" group of male eye-closed and single-legged standing with an average of 34.2 seconds, the standard deviation of 29.16; female eye-closed and single-legged standing with an average value of 28.1 seconds, the standard deviation of 27.16. The average male choice reaction time of 0.66 seconds, the standard deviation was 0.12 ; the average woman choice reaction time of 0.76 seconds, the standard deviation of 0.17 .

"Ant tribe" average male push-up group was 23.75 , standard deviation 5.76; female push-ups average value is 17.63, the standard deviation of 5.09.

The 2.4 "national physical fitness evaluation standard" standard rate

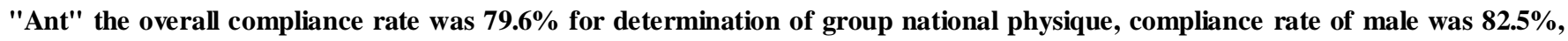
compliance rate of $76.6 \%$ for women.

3 conclusions

3.1 "Ant tribe" physique level is lower than the national physical fitness level, as Beijing city rural people's physique level. 3.2 "Ant 
tribe" population health cognition, the lack of scientific physical exercise, the poor effect of physical exercise, heart and lung function is low. 3.3 "Ant tribe" physical quality than the national physical quality, mainly for the upper limb strength and neuromuscular response ability. 3.4 "Ant tribe"group of women are trend of obese.

Keywords - the university graduates, Ant tribe, physique

\title{
都市“蚁族”群体体质健康现状的调查研究 以北京市为例
}

\author{
刘琦 \\ 北京农学院，北京，中国
}

“蚁族” 又称为大学毕业生低收入聚居群体, 指的是大学毕业后无法找到工作或工作收入很低而聚居在都市城乡结 合部的大学生。他们是继三大弱势群体（农民、农民工、下岗职工）之后的第四大弱势群体。开展本课题研究目的是更 好地了解 “蚁族” 群体体质健康变化情况, 掌握她们的群体体质特征和健康生存状况, 提供增强体质、促进健康的体育 干预措施, 让她们更好地、更快地融入社会, 成为国家发展壮大的坚实力量。同时为政府、社会了解 “蚁族” 群体的体 质健康现状提供详实资料，为我国的大众体育政策的制定提供科学依据。

\section{1. 研究对象与方法}

\section{1 研究对象}

研究选择在北京市城乡结合部和近郊农村聚居的低收 入大学毕业生为研究对象。随机选取 550 名大学毕业生, 年龄为 20 岁- - 29 岁, 其中男性 330 人, 女性 220 人。 获得有效样本 517 人, 其中男性 316 人，女性 201 人。

体质测定指标是身高、体重、肺活量、台阶试验、握 力、纵跳、仰卧起坐、俯卧撑、坐位体前屈、闭眼单脚站 立和选择反应时。

\section{2 研究方法}

本项目采用文献资料法、专家访谈法、问卷调查法、体 质测量法、数理统计法开展研究工作。主要用体质测量法 对聚居村的 550 名低收入大学毕业生进行体质测定, 获取 真实有效数据, 对数据进行统计分析、论证, 进一步认识 “蚁族” 群体体质健康水平和基本特征。主要用问卷调查 法面向聚居村低收入大学毕业生开展生活习惯、体育锻炼 情况等调查工作。

本项目研究按照国家《2010 年国民体质监测工作要求》 组织工作，采用《国民（成年人部分）体质测定标准》中 的方法和问卷对测试对象进行测试与评价, 测试的各项指 标数据应用 SPSS12.0 for Windows 软件进行统计分析, 结
果以平均值、标准差或百分率来表示。

本项目研究体质测定工具选择 “健民” GMCS-II 型体 质测试仪器。

\section{2. 研究结果与分析}

2.1 “蚁族” 群体与 2010 年全国国民体质监测同年龄组身 体形态的比较

“蚁族”群体与 2010 年全国国民体质监测 20-29 岁年 龄组身体形态比较结果显示, “蚁族” 男性、女性身高平 均值分别低于同年龄组国民男、女身高 0.94 厘米和 2.2 厘 米, 检验结果表示差异非常显著 $(P<0.01)$ 。“蚁族” 男 性体重平均值低于同年龄组国民男性 3.02 公斤，检验结 果表示差异非常显著 $(P<0.01)$; 女性体重平均值高于 0.22 公斤, 检验结果表示差异不显著 $(P>0.05)$ 。从身 高和体重结果分析 “蚁族” 男性、女性身高和男性体重均 低于同年龄组国民体质, 这可能与样本来源有关。样本大 多数来源于全国各地的偏远地区和农村, 地区与家庭经济 落后, 从小营养不良, 对身高、体重会产生一定的影响; 来京后经济收入也非常低, 经常以一些 “垃圾” 食品为伴 等, 对体重会产生一定的影响。“蚁族” 女性体重稍高于 同年龄组女性国民体质, 但差异非常不显著, 这可能与样 本抽取有关, 也与其爱吃零食、饮食不均和缺少体育锻炼 
有关。问卷调查结果显示 “蚁族” 群体每周不参加体育锻 炼的占 $20 \%$, 每周参加一次体育锻炼的占 $24 \%$, 而 $50 \%$ 参加 体育锻炼的人锻炼时间不超过 30 分钟, 这种体育锻炼方式 对身高不会有什么影响, 但对其体重会有较大影响。

2.2 “蚁族” 群体与 2010 年全国国民体质监测同年龄组身 体机能的比较

“蚁族” 群体与 2010 年全国国民体质监测 20-29 岁年 龄组身体机能比较结果显示, “蚁族” 群体男性、女性肺 活量、台阶试验平均值均低于同年龄组国民男、女。其中, “蚁族” 群体男性肺活量低 72 毫升, 检验结果差异显著 ( P $<0.05)$; 男性台阶试验低 6.39, 检验结果差异非常显著 $(\mathrm{P}<0.01)$; “蚁族” 群体女性肺活量低 130 毫升, 检验 结果差异非常显著 $(P<0.01)$; 女性台阶试验低 4.5, 检 验结果差异非常显著 $(\mathrm{P}<0.01)$ 。从结果。

分析 “蚁族” 群体男性、女性肺活量和台阶试验均低 于同年龄组国民体质, 这可能与样本是否参加体育锻炼和 体育锻炼的方式有关。问卷调查结果显示样本闲暇时间主 要用于游玩 (26\%) 和视听娱乐 (21\%), 而用于体育锻炼 的时间仅占 $9 \%$; 并且这些体育锻炼采用的方式多为步行 （29\%）和跑步（21\%）; 即使是步行和跑步的体育锻炼所 用的时间有 $50 \%$ 的人在 30 分钟以下, 这种较少的运动时间 是达不到进一步增强体质的目的。可见 “蚁族” 群体平时 主动地、有计划地参加体育锻炼的时间很少, 参加体育锻 炼的项目也多是选择强度较低, 负荷较小的运动项目, 而 在体育锻炼中感觉呼吸、心跳变化不大者为 $16 \%$, 呼吸、 心跳略有变化和微微出汗者 $67 \%$, 显然体育锻炼的效果不 尽人意, 这可见 “蚁族” 群体缺少科学健身的常识, 缺少 科学健身的指导。正如问卷调查结果显示的那样, “蚁族” 群体参加体育锻炼的第一目的是减轻压力, 缓解情绪。这 说明 “蚁族” 群体身体机能低下的主要原因是缺少科学的 体育锻炼。

2.3 “蚁族” 群体与 2010 年全国国民体质监测同年龄组身 体素质比较

“蚁族” 群体与 2010 年全国国民体质监测 20-29 岁年 龄组身体素质比较结果显示, “蚁族” 群体男性和女性的 握力、纵跳、坐位体前屈、闭眼单脚站立、俯卧撑、一分 钟仰卧起坐指标平均值均低于同年龄组国民男、女, 选择 反应时指标平均值高于同年龄组男女。其中男性纵跳、闭 眼单脚站立和男女坐位体前屈差异不显著, 其它均差异非 常显著。
“蚁族” 群体身体力量素质的握力、纵跳、俯卧撑、 一分钟仰卧起坐指标平均值均低于同年龄组国民身体力量 素质的主要原因是其参加的体育锻炼内容中缺少力量性方 面的锻炼。问卷调查显示这些正处在身体强壮期的人们把 “步行” 作为第一位体育锻炼项目, 而能够主动参加力量、 健美体育锻炼的仅占 $3 \%$ 。

“蚁族” 群体男女的选择反应时均高于同年龄组国民 男女反应时, 差异非常显著。问卷调查显示 “蚁族” 群体 经常参加球类体育锻炼的仅占 $12 \%$, 参加武术类的体育锻 炼仅有 $1 \%$; 从事的工作主要是专业技术人员（32\%）和商 业、服务业人员（20\%）。这些说明了该群体缺少影响神经 肌肉反应速度和动作综合能力的体育锻炼, 或者说也缺少 影响神经肌肉反应速度和动作综合能力的工作。

“蚁族” 群体男女的闭眼单脚站立指标均低于同年龄 组国民男女, 女性差异非常显著, 男性差异不显著, 这可 能与男性较之女性从事体力劳动较多有关。“蚁族” 群体 身体柔韧素质的坐位体前屈指标均低于同年龄组国民, 但 差异不显著, 这可能与人们的简单身体活动习惯一一喜欢 伸伸腰、屈屈体等有关。

2.4 “蚁族” 群体与 2010 年全国国民体质监测达标率的比 较

达标率是指国民达到《国民体质测定标准》合格等级 以上比例。研究结果显示, “蚁族” 群体男性合格率高于 女性, 良好率、优秀率低于女性, 而不合格率女性高于男 性。

“蚁族” 群体达不到合格标准的主要指标为选择反应 时和握力, 说明该群体上肢力量不足明显, 神经肌肉联系 迟缓。

研究结果显示, “蚁族” 群体体质测定总体达标率比 2010 年全国达到《国民体质测定标准》 “合格” 以上标准 的人数比例低 9.3 个百分点; 男性比 2010 年全国男性达到 《标准》 “合格” 以上标准的人数比例低 5.8 个百分点; 女性比 2010 年全国女性达到《标准》 “合格” 以上标准的 人数比例低 12.8 个百分点, 差异均非常显著。

研究结果显示, “蚁族” 群体体质测定总体达标率比 2010 北京市达到《国民体质测定标准》 “合格” 以上标准 的人数比例低 10.8 个百分点, 比城镇人群低 15.5 个百分 点, 差异均非常显著; 比乡村人群低 3.3 个百分点, 差异 显著。

“蚁族” 群体总体、男性和女性体质达标率均低于 2010 年全国总体、男性和女性, 也低于全国及北京市城镇 
或乡村人群。究其原因主要是该群体主要来自全国各地的 偏远乡村; 生活上无安定的居所而群居, 工作上多为商业、 服务业的工作而收入微薄，压力远远超过北京市的村民; 缺少体育锻炼和科学的指导。

\section{3. 结论与建议}

3. 1 “蚁族”群体体质总体水平低于目前国民体质水平, 不如北京市乡村人群体质水平。建议加强 “蚁族” 群体在 北京生活、工作的相关政策制度建设。

3.2 “蚁族” 群体体质健康认识模糊, 缺少科学的体育 锻炼, 体育锻炼效果较差, 心肺机能较低。建议加强学校 阶段的体质健康教育, 尤其在大学阶段, 使他们在迈出校 门走进社会前就形成正确的体质健康观念, 并具备自我科 学健身的能力。

3. 3 “蚁族” 群体身体素质不如国民身体素质, 主要 表现为上肢力量和神经肌肉反应能力较差。提倡该人群多 参加综合性强的体育锻炼, 加强有针对性的力量锻炼。

3. 4 “蚁族” 群体女性有肥胖倾向。加强体育锻炼减肥 是适合该群体的好方法; 改变不良生活饮食习惯是关键; 解决安居生活是重点。

3.5 从宏观上看, “蚁族” 的形成是社会发展中的问
题, 只有化解这一矛盾, 其体质健康问题才会迎刃而解。

\section{参考文献(References)}

[1] Lian. Ant -- University Graduate Village Inhabited record. Guilin: Guangxi Normal University press, 2008.09

[2] State Administration of Sports. In 2010 the national physique monitoring bulletin [EB]: (2011-09-02) (2011-10-10) http://www.sport.gov.cn/n16/index.html

[3] State Administration of Sports. Beijing: People's sports press, 2003.08

[4] State Administration of Sports, Chinese national physique monitoring system research group. Research Chinese national physique monitoring system. Beijing: Beijing Sport University press, 2000.08.

[5] Jiang Chongmin. The national physical fitness evaluation standard knowledge. Beijing: China Standard Press, 2007

[6] the State General Administration of sports. The second national physique monitoring report. Beijing: People's sports press, 2007 